\title{
An Automated Method for Characterization of Evoked Single Trial Local Field Potentials Recorded from Rat Barrel Cortex under Mechanical Whisker Stimulation
}

\author{
Mufti Mahmud · Claudia Cecchetto · Stefano Vassanelli
}

Received: 29-02-2016/ Accepted: date

\begin{abstract}
Rodents explore their surroundings through whisking by localizing objects and detecting textures very precisely. During such tactile exploration, whisker deflection is first mechanically transduced by receptors and then information encoded throughout the somatosensory pathway ending in the somatosensory 'barrel' cortex. In the barrel cortex, tactile information from a single whisker is segregated and processed in a cortical column corresponding to the deflected whisker. Local Field Potentials (LFPs) generated by whisker deflection in the barrel cortex present typical signatures in terms of shape and amplitude that are related to the activation of the local neuronal populations. Therefore, rigorous analysis of such responses may reveal important features about the function of underlying neuronal microcircuits. In this context, software methods for characterizing single-trial LFPs are needed that are also suitable for online extraction of LFP features and for brain-machine interfacing applications. In this work we present an automated and efficient method to analyze evoked LFP responses in the rat barrel cortex through automatic removal of stimulation artifacts, detection of single events and characterization of their relevant parameters. Evoked single-trial LFPs recorded under two different anesthetics are examined to demonstrate the feasibility, accuracy and applicability of the method.
\end{abstract}

Mufti Mahmud (ه)

NeuroChip Lab, Department of Biomedical Sciences, University of Padova, 35131 - Padova, Italy

E-mail: muftimahmud@gmail.com

Claudia Cecchetto

NeuroChip Lab, Department of Biomedical Sciences, University of Padova, 35131 - Padova, Italy

Department of Information Engineering, University of Padova, 35131

- Padova, Italy

Stefano Vassanelli

NeuroChip Lab, Department of Biomedical Sciences, University of Padova, 35131 - Padova, Italy
Keywords Neuronal signal processing - neuronal signal analysis $\cdot$ barrel cortex $\cdot$ local field potentials $\cdot$ whisker stimulation

\section{Introduction}

Whisking is a dominant contributor of sensory information in rodents. Through whisking rodents are capable of localizing objects, detect textures and differentiate shapes very precisely [1]. Whiskers in the rodent snout are represented in a cortical region called 'barrel cortex' following a simple oneto-one topographic mapping where single columns correspond to single whiskers. As such, the somatosensory pathway related to whisking is a valuable prototype system to study information processing of sensory inputs in rodents [2]. Yet, understanding how neuronal networks in the barrel cortex represent information on whisker deflection remains a largely unmet challenge [3]. Intracellular single neuron recording (e.g., patch-clamp) can only partially contribute to our understanding of the network and must therefore be complemented by experimental strategies to investigate neuronal populations. Thus, among other available techniques, the recording of population Local Field Potentials (LFPs) is of primary importance [4]. While it is recognized that network function can be in part inferred from LFPs, not much has been done for developing software tools for their automated analysis, which is still mainly delegated to manual operation [5].

Stimulus evoked LFPs in the barrel cortex are thought to mainly derive from activity of neuronal populations in the vicinity of the recording electrode and can therefore be considered as fingerprints of local network activity [6]. For this reason, it is a general practice to repeatedly record singletrial LFPs (i.e. responses to a given stimulus) and then obtain a stimulus-locked averaged signal to be characterized under 
the assumption that the network response to the same input is similar across trials. However, this may be a too strong assumption. Previous studies have demonstrated that relevant information provided by single-trial LFPs may be lost in the averaged signal [7,8]. Consequently, extraction of singletrial LFP paramenters can be important to truly characterize the network dynamics (e.g., by determining the cortical layer activation order [5]) and to reconstruct neuronal activity (e.g., by current source density analysis). In this context, signal shape characterization also plays a fundamental role [9] and methods are required to extract essential shape features and to cluster single-trial LFPs accordingly. Whereas a number of methods are available to perform a detailed characterization of neuronal spikes [10], methods for LFPs analysis are lagging behind. Recent efforts have been made contributing to fill the gap [11,12,13] including denoising [14], artifact removal [15,16], and shape based classification of LFPs [17, 18]. Efficient automated methods for single-trial LFPs characterization, however, are still lacking. This represents a challenge given the typical variability of shapes that can be found among single-trial LFPs when recording in the barrel region [5] as well as by the various possible contaminations from stimulus artifacts.

In this work, we present an automated and efficient tool to characterize single-trial LFPs recorded in the barrel cortex upon whisker deflection. The characterization is mainly done in two passes - (1) In the first pass, the individual signal files are preprocessed (e.g., scanned for the presence of any stimulus artifacts and if found, removed automatically); (2) In the second pass, the evoked responses are identified and characterized (e.g., by detecting important shape features and calculating relevant parameters of the responses). The identified features and estimated parameters include: the response-onset (start of the evoked response), the responseonset latency (time delay between the stimulus-onset and the start of the response), the response peak (apex of the response), the response-peak latency (time delay between the stimulus-onset and the apex of the response), the event amplitude at the response peak, and the positive rebound (the apex of the positive rebound after the response peak). At the end, these information are automatically written in an American Standard Code for Information Interchange (ASCII) coded text file, and necessary figures are generated and reported to the user.

We report that the automatic feature detection and parameter estimation yielded similar results in comparison to manual calculation performed by hand. Finally, as an example of the tool's usability, we report a preliminary characterization of single-trial LFPs variability depending on two different types of anesthesia.

\section{Methods}

\subsection{Signal Acquisition}

The single trial LFPs were recorded from Wistar rats which were maintained in the Animal Research Facility of the Department of Biomedical Sciences (University of Padova, Italy) under standard environmental conditions. The University of Padova Ethical Committee approved all animal procedures. The data acquisition procedure was followed as per the description in [19]. The experimental setup is seen in Fig. 1]

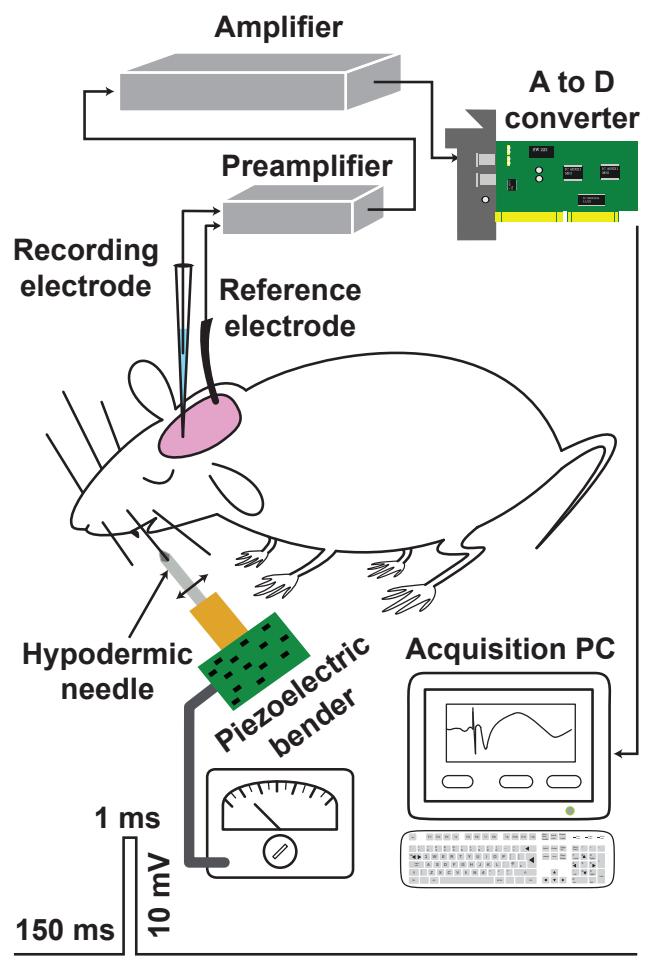

Figure 1 Acquisition of evoked LFP responses from rat barrel cortex (S1) upon whisker deflection. The arrow on the hypodermic needle, attached to the piezoelectric bender, shows the direction of its movement. The stimulus is shown at the bottom which is used in driving the piezoelectric bender for dorsoventral movement of the whisker inserted in the needle. The indicated $10 \mathrm{~V}$ trigger impulse produced by the waveform generator was translated to $60 \mathrm{~V}$ and applied to the piezoelectric bender for the required amount of displacement.

\subsubsection{Surgical Procedures}

P30-P50 rats were anesthetized with a mixture of Tiletamine and Xylazine (Til-Xyl) or Urethane. The anesthesia level was monitored throughout the experiment by checking reflexes, respiration and the absence of whiskers' spontaneous movements. Additional doses of anesthetics were administered to maintain a constant anesthesia level. During the surgery and recording section, animals were kept on a stereotaxic apparatus under a stereomicroscope and fixed by 
teeth and ear bars. The body temperature was monitored with a rectal probe and maintained at $37{ }^{\circ} \mathrm{C}$ using a heating pad. An incision was made along the medial line of the head, starting from the eyeline and ending at the neck. Thus, the skull over the right hemisphere was drilled to open a window in correspondence of the somatosensory cortex, S1 (-1 to $-4 \mathrm{AP},+4$ to $+8 \mathrm{ML}$ ) [20]. Meninges were then carefully cut by means of forceps at coordinates $-2.5 \mathrm{AP},+6 \mathrm{LM}$ for the insertion of the recording micropipette. Throughout all surgical operations and recordings, the brain was bathed by standard Krebs solution kept at $37^{\circ} \mathrm{C}$.

\subsubsection{Cortical Laminar Electrodes}

Evoked extracellular LFPs generated throughout the layers of the barrel cortex were recorded and analyzed. In these experiments, recordings were performed at $100 \mu \mathrm{m}$ resolution by means of conventional $\mathrm{Ag} / \mathrm{AgCl}$ electrodes inserted into borosilicate glass micropipettes (GB150T-10, Science Products $\mathrm{GmbH}$, Hofheim, Germany) with tip diameter $\sim 1 \mu \mathrm{m}$ and resistance $\sim 1 \mathrm{M} \Omega$, starting from Layer II $(320 \mu \mathrm{m})$ down to Layer Va $(920 \mu \mathrm{m})$. The micropipette was inserted into a holder connected to a PatchStar micromanipulator (Scientifica, East Sussex, UK) for fine control of the electrode during the experiment.

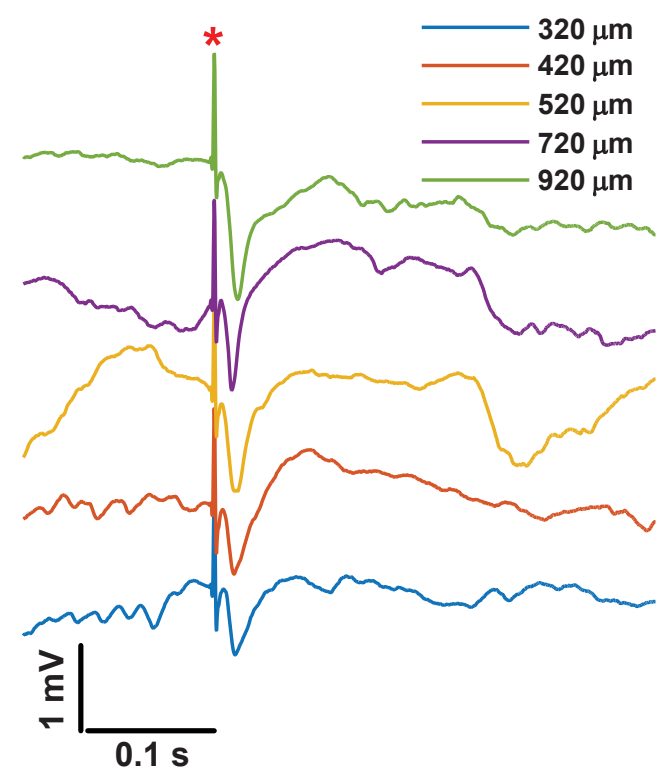

Figure 2 Representative examples of single trial LFP responses to whisker deflection from five different depths under Til-Xyl anesthesia. The red ' $*$ ' at the top indicates the stimulus artifact.

\subsubsection{Whisker Stimulation Protocol}

After craniotomy, contralateral whiskers were trimmed at about $10 \mathrm{~mm}$ from the mystacial pad. The principal whisker was then identified, as the one which provided the maximum response amplitude when deflected, from a subset of whiskers corresponding topologically to the electrode's position in the cortex. The principal whisker was deflected repeatedly by rapidly displacing a $25 \mathrm{G}$ hypodermic needle (BD Plastipak, Madrid, Spain) which was attached to a piezoelectric bender (P-871.122, Physik Instrumente, Karlsruhe, Germany) using double-sided tape. The bender was driven by a waveform generator (Agilent 33250A $80 \mathrm{MHz}$, Agilent Technologies Inc., Colorado, USA) by providing square stimuli at $1 \mathrm{kHz}$ which was triggered by a custom LabView program (www.ni.com/labview/).

\subsubsection{Recording Cortical Laminar Profiles of Evoked LFP Responses}

The LFP responses evoked after whisker deflection were acquired by the recording electrode with reference to a ground electrode (see Fig. 1). Both electrodes were connected to an extracellular preamplifier or head-stage (SEC-EXT, npi electronic $\mathrm{GmbH}$, Tamm, Germany) having $10 x$ gain and high-pass filter with corner frequency of $1 \mathrm{~Hz}$. The preamplified waveforms were then $10 x$ amplified and low-pass filtered with cutoff frequency of $33 \mathrm{kHz}$ using an amplifier (SEC-10L, npi electronic GmbH, Tamm, Germany) operating in bridge mode. The amplified raw signals were stored by an acquisition PC at $50 \mathrm{kHz}$ sampling rate using NI PCI6071E data acquisition board (National Instruments Inc, Texas, USA) combined with a BNC-2010 terminal block (National Instruments Inc, Texas, USA).

At each cortical depth 500 single trial LFP responses were recorded in response to the mechanical deflection of the principal whisker. A temporal delay of 2 seconds was set between subsequent traces to avoid any phenomenon related to adaptation. A depth profile resulting from an experiment is seen in Fig. 2.

\subsection{Software Development}

A multi-layered software engineering approach was adopted in designing the software with three layers as shown in Fig. 3.

In this type of client-server architecture user interface, functional process logic ('business logic'), and data storage and access are maintained as independent modules to promote software modularity allowing any of the layers to be upgraded or replaced independently with changing requirements [21].

This tool was designed with the following three layers:

- User interface layer (top layer): This contained user interfaces which, upon receiving user commands, communicated with the middle layer for their execution. 


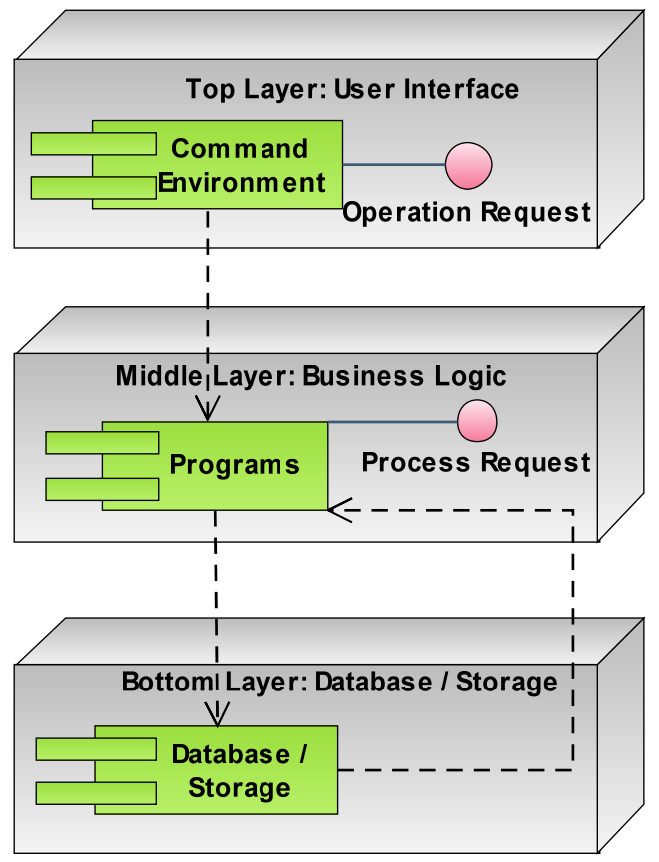

Figure 3 Three-layered architecture of the software.

- Application layer (middle layer): The detailed processing and analysis algorithms were implemented in this layer. It was designed to be capable of communicating and exchanging data between the other two layers.

- Data layer (bottom layer): Storages (or databases) were kept as a separate layer to improve scalability, data security, and performance.

The algorithms were implemented in MATLAB (R2015b, Mathworks Inc., Natick, USA) under Windows 8.1 environment (Microsoft Inc., Redmond, USA).

The tool will be made available online under the GNUGPL license as a part of the 'SigMate' software package [12, 22, 23].

\subsubsection{Stimulus Artifacts Removal}

The artifact removal algorithm was divided into two main subfunctions: Artifact Detection (AD), and Artifact Removal (AR). The AD subfunction scanned each signal for occurrence of artifacts and if found, marked the probable end of the detected artifacts. The AR subfunction calculated the artifacts' exact initiation and end points, removed the artifacts, and interpolated the artifact region. Once the artifact was removed, the clean signal (see Fig. 6) was stored for further processing and analysis. The artifact removal process is shown as a flowchart in Fig. 4.

The following two subsections describe the algorithms for $\mathrm{AD}$ and $\mathrm{AR}$ in greater details.

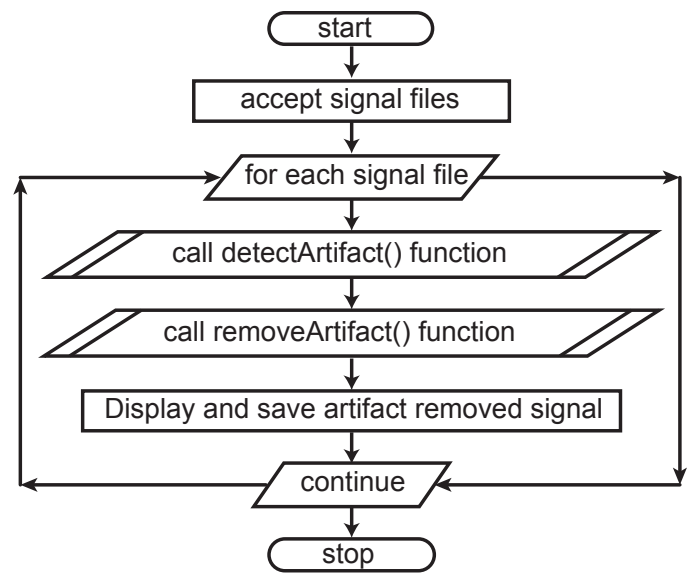

Figure 4 Flowchart of the artifact removal method.

Artifact Detection (AD) This subfunction provided with the number of artifacts present in a single trial LFP signal and marked the probable end of the artifacts' which was then sent to the next module for removal. To detect an artifact each data point of the signal starting from the stimulus-onset was checked for threshold crossing and its direction with respect to the previous data-point (up or down). When a pair of threshold crossing data points with same directions was detected in the signal, the second point in the pair was considered as a probable end of an artifact. The pseudocode is listed in Algorithm 1.

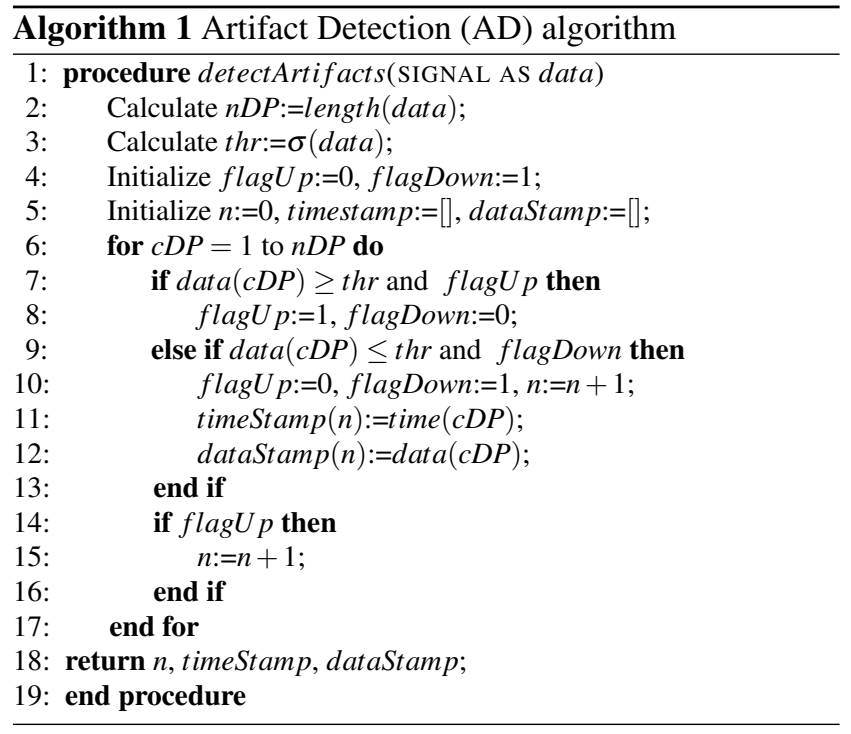

Artifact Removal (AR) Once the information on the number of artifacts and their probable end points were determined, this module was implemented to determine the exact start and end of those artifacts. To calculate the exact area covered by the artifacts, the probable end point received from $\mathrm{AD}$ 
subfunction was taken as reference. The signal was then scanned backwards from the reference point and the local minima was computed. Starting from the detected local minima where the signal derivative approached zero was taken as the starting point of the artifact. For the exact end of the artifact it was sufficient to detect the point nearest in value to the starting point. Thus, to calculate the end point: from the reference point the signal was scanned forward, the difference in derivative between the starting point and current point was calculated, and the process continued until this difference crosses a predefined error value (defined as $10 \%$ of the signal standard deviation). Once the starting and ending points were detected, interpolation between them was performed, and the artifact area was replaced with the interpolated points. The pseudocode is listed in Algorithm 2

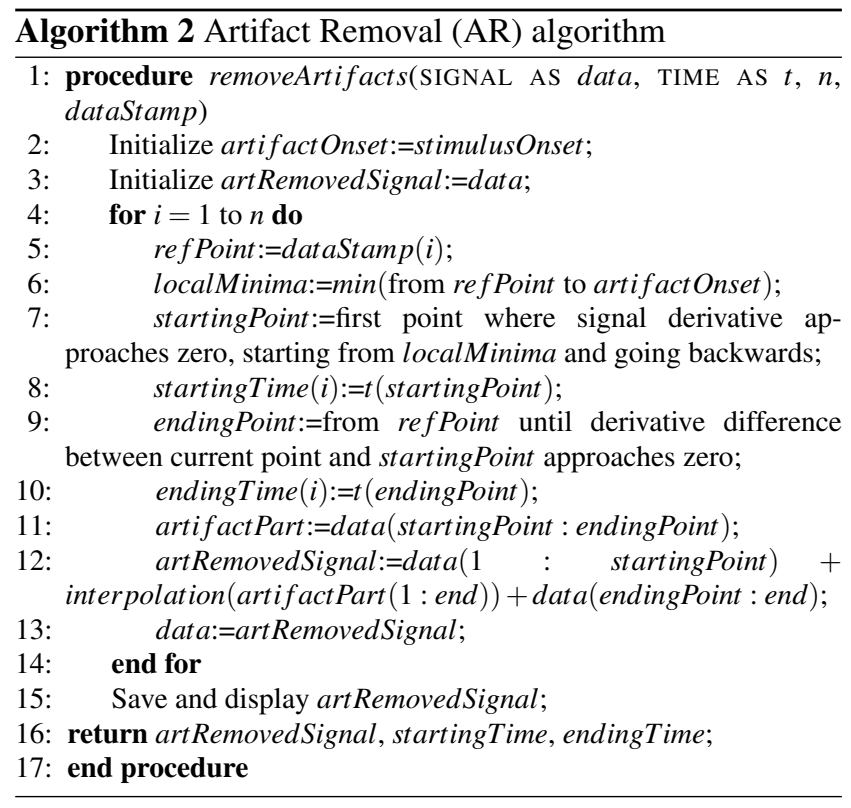

\subsubsection{Event Characterization}

The Evoked Response Evoked responses from the barrel cortex follow a specific laminal morphology which has been reported earlier [24,25]. Usually in upper cortical layers (I, II) the response rarely have a small positive peak (SP) followed by the main negative peak, i.e., the response peak (RP) and then a positive rebound (PR). In the mid-layers (III, IV, and V) the SP disappears and the signals start with the RP followed by the PR (see Fig. 5). In deep cortical layers (VI), the main RP becomes smaller and usually gets divided into two smaller negative peaks followed by the PR. These features of the signals can be exploited to automatically detect the evoked responses in the recorded signals. Overall, heuristically, the evoked responses are characterized by three features (see Fig. 5): the start of the response (response-onset or Feature 1), the RP (Feature 2), and the PR (Feature 3). Fig. 5 shows an annotated example of a single trial LFP response recorded from layer IV $(720 \mu \mathrm{m})$ where the features are highlighted [5]. It should be noted that in cases where the SP was detected, the response-onset or Feature 1 was considered as the maxima of SP. In the remaining text, the terms Feature 1 and response-onset are used interchangeably.

Accordingly, as it has been reported earlier [5], three corresponding parameters are used to characterize the evoked responses: response onset latency $(R O L)$, response peak latency $(R P L)$, and response peak amplitude (RPA). However, to be able to compute these parameters precisely, accurate detection of the related features is very important and a custom algorithm was developed to the purpose.

Feature Detection and Parameter Calculation The following algorithm (Algorithm 3) detected features in evoked responses, by scanning artifact removed single trial LFPs.

As shown in the Algorithm 3 each single trial LFP was lowpass filtered $(250 \mathrm{~Hz}$, butterworth lowpass filter) and translated (on the Y-Axis) by setting the signal amplitude at the stimulus-onset to zero. This translation helped to avoid the slow deviation of signal that might obscure the real amplitude of the features. Then the second derivative of the signal was obtained to facilitate the detection of features representing the evoked response by evaluating the signal shape change in the response area. Large fluctuations in the derivative were identified which correspond to the signal shape change and thus were used in detecting the features.

The algorithm first identifies the response-onset as the starting point of the evoked response. For the accurate identification of response-onset, each point from the stimulusonset was checked for a threshold-crossing. The standard deviation of the signal's steady-state part (i.e., the signal part before stimulus-onset) was taken as threshold. Signal of 10 ms duration after the stimulus-onset were divided into small parts of $0.5 \mathrm{~ms}$ and derivatives of those parts were obtained. The response-onset was identified as the time instance of the signal when the small parts derivative was found to exceed \pm threshold value.

After detection of the response-onset the rest of the signal was divided into chunks and scanned for derivative change to find the other features. Care was taken in case of the SP, which is rarely present in a signal and if present, may have either positive or negative direction. In case of the positive direction, a threshold of $10 \mu \mathrm{V}$ was set to make sure that it indeed was a feature and not just background spontaneous brain activity. If the signal was found to be going down, then the maximum negative peak was found and from that peak a time window $\pm 5 \mathrm{~ms}$ was selected and scanned for occurrence of yet another negative peak. If a second negative peak was detected, the first negative peak was set as Feature 1 


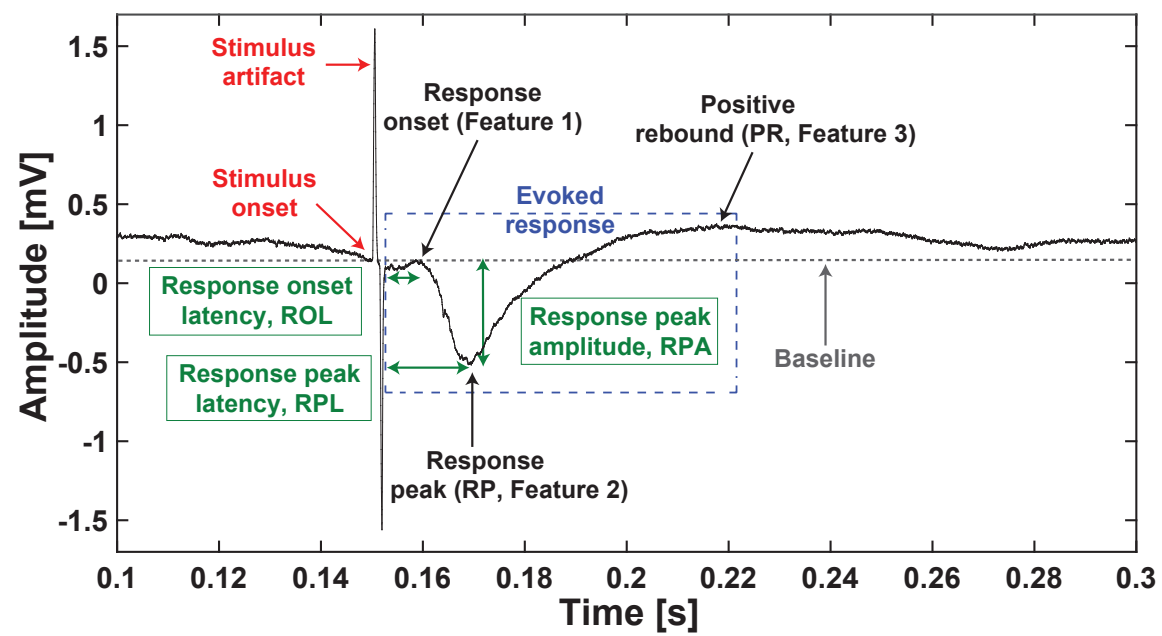

Figure 5 Representative single trial LFP with relevant features and parameters. The grey straight line indicates the baseline. The blue dashed box shows the annotated evoked response with its different features (in black) and defining parameters (in green).

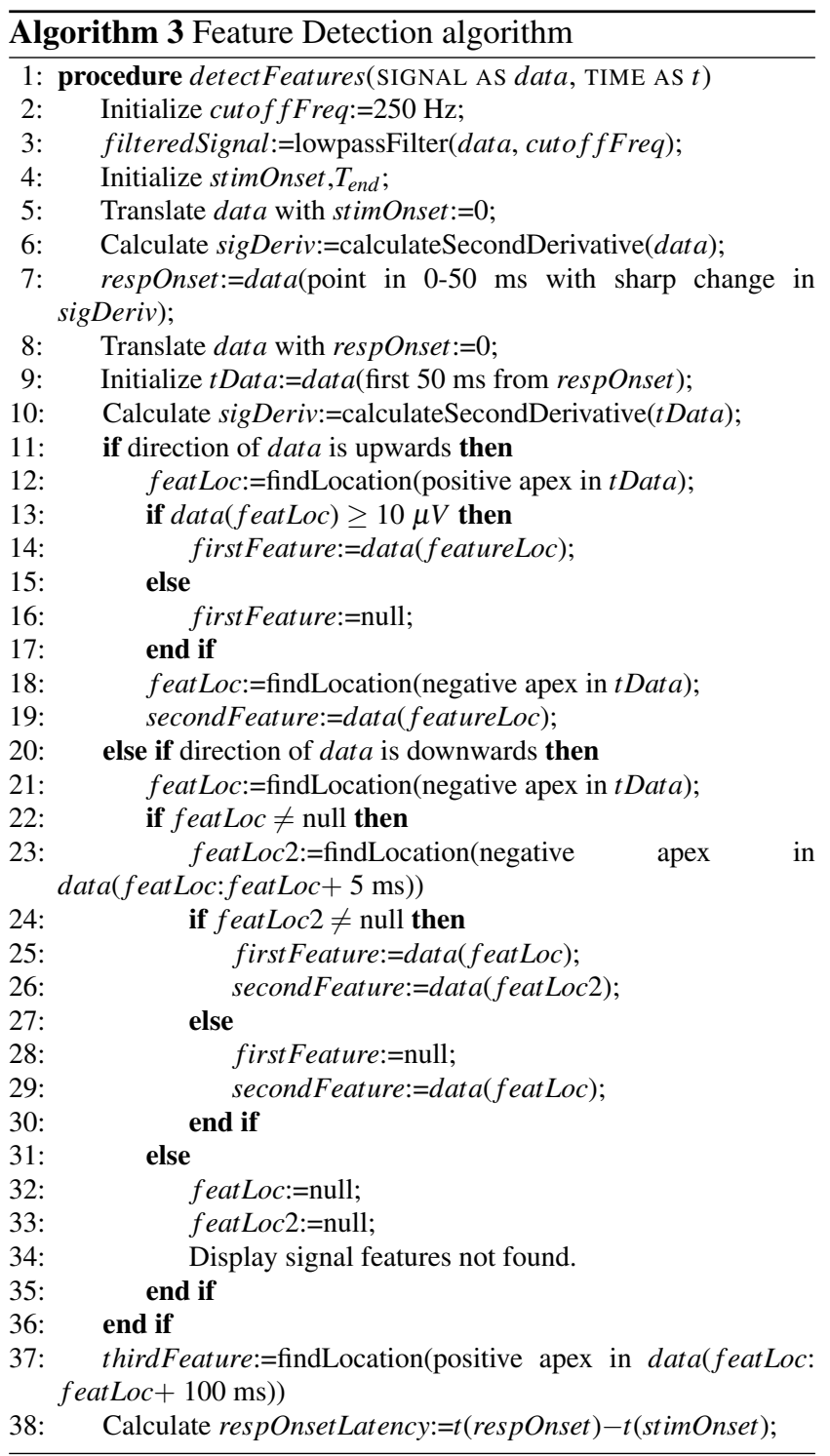

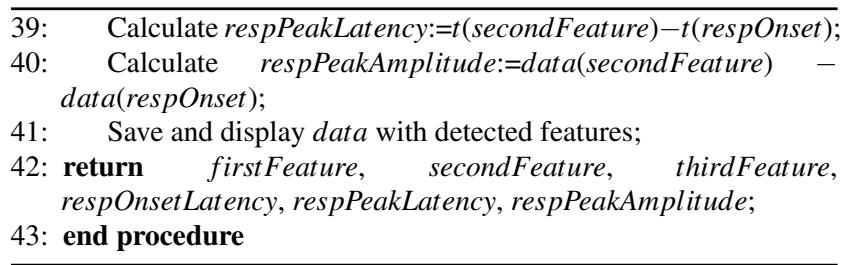

and the second peak as Feature $2(R P)$; otherwise, the positive peak was absent and the Feature $2(R P)$ was set as the maximum negative peak

The Feature $3(P R)$ was set as the positive apex in the time window between Feature $2(R P)$ and next $100 \mathrm{~ms}$.

Parameter Calculation Once the features (Features 1,2,3) were detected the important parameters $(R O L, R P L$, and $R P A)$ were estimated as follows:

$R O L=$ Time (response-onset $)-$ Time(stimulus-onset)

$R P L=$ Time(Feature 2) - Time (response-onset)

$R P A=$ Amplitude(Feature 2)-Amplitude(Feature 1)

in where the functions Time() and Amplitude() denotes the instantaneous time and amplitude of the signal at a given point. The detected features and estimated parameters were saved in a file for further processing.

\section{Results and Discussion}

The tool was tested on single trial LFP responses recorded from rat barrel cortex evoked by whisker deflection under a mixture of Til-Xyl or Urethane anesthesia. As evidentiated in the subsequent sections, the tool successfully removed stimulus artifacts, detected different features of the evoked response, and accurately estimated the signal shape characteristics. Also the tool has an option to perform batchprocessing of multiple experiments. 


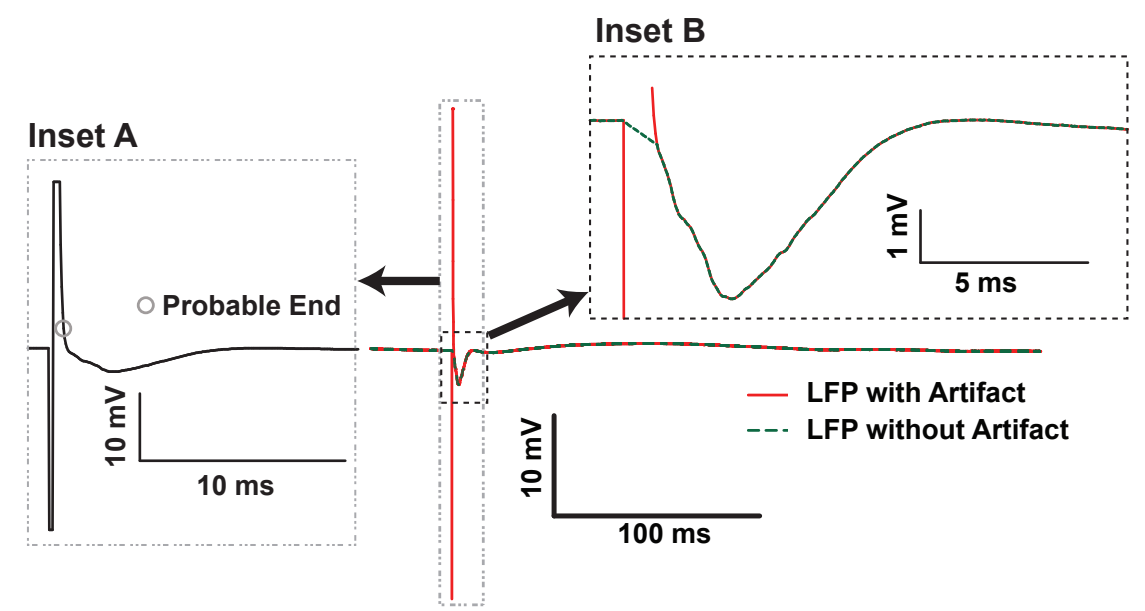

Figure 6 Outcome of the artifact detection and removal process in a representative single trial LFP response. The red trace is with artifact and the green one is without artifact. Inset A shows the probable artifact ending point as an outcome of the artifact detection process. Inset B shows the signals before and after the artifact removal process.

\subsection{Artifact Detection and Removal}

Fig. 6 shows the result of the artifact detection and removal algorithm on an example of a single trial LFP response. At first the artifact detection process detected the probable end point of the artifact (Fig. 6, Inset A) which was then used in detecting the artifact's start and end more precisely and at last removed from the signal. It's worth mentioning that the single trial LFP response shown in Fig. 6 was with only one stimulation artifact present, but the algorithm is capable of successfully removing multiple stimulation artifacts from single trial LFPs in one run.

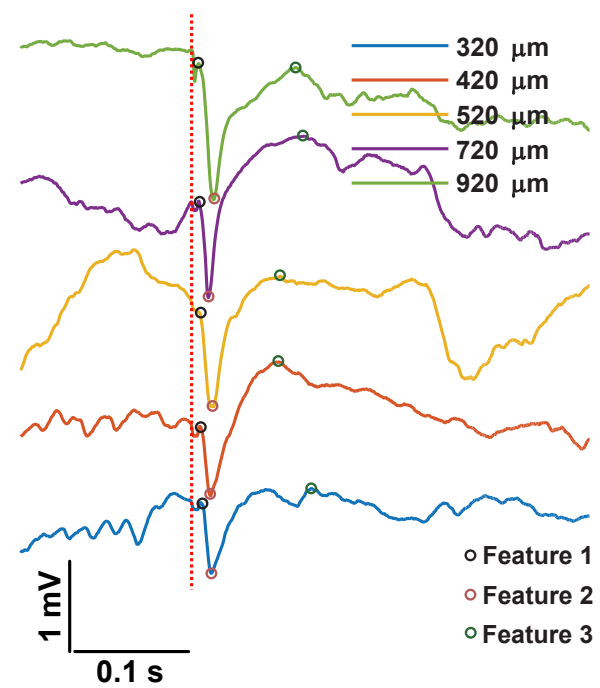

Figure 7 Artifact removed depth profile of representative single trial LFPs corresponding to the traces shown in Fig. 2 The small circles denote the detected features in the artifact removed evoked responses using the feature detection algorithm (Algorithm 3 . The vertical dashed line in red represents the stimulus - onset.

\subsection{Feature Detection and Parameter Estimation}

The feature detection algorithm performed accurate detection of the important features defining the evoked responses in the single trial LFPs. Fig. 7 illustrates an example of the algorithm's single run on a representative dataset. From 500 single trial LFPs recorded and analyzed for each cortical depth, one trace per depth was randomly selected for demonstration purpose. It is also seen in Fig. 7 that the features defining the evoked responses in the single trial LFPs were detected precisely by the feature detection algorithm.

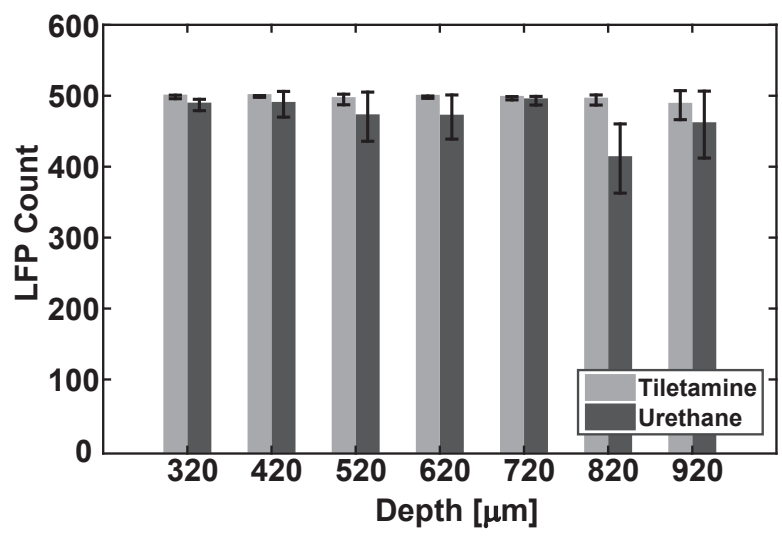

Figure 8 Feature detection accuracy in the single trial LFPs recorded under two different anesthetics (a mixture of Til-Xyl, and Urethane). Means and \pm standard deviations are represented by bars and error bars, respectively. The means and standard deviations were obtained with at least three experiments $(n=3)$ from each cortical depth.

Fig. 8 highlights the accuracy of the feature detection in the single trial LFP evoked responses under two anesthetics (a mixture of Til-Xyl, and Urethane). It can be seen that the 
responses recorded under tiletemine-based anesthetic were more similar to the usual evoked response's signature ( $99.06 \%$ of recorded single trial LFPs were detected) in comparison to the ones under Urethane-based anesthetic ( $93.69 \%$ of recorded single trial LFPs were detected).

To judge the accuracy of parameter estimation by the proposed automatic method of features detection, the parameters were compared to the ones computed manually. This manual computation of parameters was done using pClamp software (version 10, Molecular Devices LLC., Sunnyvale, USA; WW.moleculardevices.com/) by annotating the signal features and employing the relations given in 'Parameter Calculation' paragraph (see Sec. ). As reported in Table 1. the automatically estimated values of the parameters are very similar to the manually estimated ones. In that table, the stated values were calculated from a set of representative single trial LFPs recorded from different cortical depths. As a further verification, the number of single trial LFPs were increased, parameters were automatically estimated and root mean square error (RMSE) were calculated between the automatically and manually estimated values. A very low RMSE value $(\sim 0.051)$ indicated that the calculation of parameters using the automated method was accurate. For the sake of simplicity in representation, values reported in Table 11 are from single trial LFPs recorded during a single experiment under the Til-Xyl anesthesia. The parameter estimation performed equally well in the recorded single trial LFPs regardless of the anesthesia used.

Table 1 Comparison of manual and automatic estimation of the parameters defined in sec. 2.2 .2

\begin{tabular}{ccccc}
\hline \hline \multirow{2}{*}{ Depth } & Mode & $R O L(\mathrm{~ms})$ & $\begin{array}{c}\text { Parameters } \\
R P L(\mathrm{~ms})\end{array}$ & $R P A(\mathrm{mV})$ \\
\hline \hline \multirow{2}{*}{$320 \mu \mathrm{m}$} & Manual & 8.72 & 19.71 & -0.90 \\
& Auto & 8.42 & 19.58 & -0.85 \\
\hline \multirow{2}{*}{$420 \mu \mathrm{m}$} & Manual & 8.04 & 17.82 & -1.70 \\
& Auto & 7.82 & 18.08 & -1.66 \\
\hline \multirow{2}{*}{$520 \mu \mathrm{m}$} & Manual & 7.16 & 15.46 & -1.02 \\
& Auto & 7.54 & 15.08 & -1.00 \\
\hline \multirow{2}{*}{$720 \mu \mathrm{m}$} & Manual & 6.7 & 16.50 & -1.65 \\
& Auto & 6.94 & 16.68 & -1.62 \\
\hline \multirow{2}{*}{$920 \mu \mathrm{m}$} & Manual & 5.96 & 18.88 & -1.60 \\
& Auto & 5.70 & 18.70 & -1.61 \\
\hline \hline
\end{tabular}

\subsection{Detecting Anesthesia Effect in single trial LFP Variability}

To demonstrate the usefulness of the tool it was applied to estimate the single trial LFP responses' variability under two different anesthetics, either Til-Xyl or Urethane. The representative features from hundreds of single trial LFP responses were detected and characterized, and the necessary parameters were estimated. The effect of the anesthesia on the sens- ory information processing was assessed by comparing the main parameters of the evoked single trial LFPs (see Fig. 5) recorded from different rats $(n=9)$. Distributions were calculated of the extracted parameters from the evoked single trial LFP responses. To better illustrate the trends in each anesthetic, estimation of the histograms were calculated by fitting higher order polynomials. Data from different rats were binned together: 5 rats in the case of Til-Xyl anesthetic and 4 rats in the case of Urethane anesthetic. The detected principal events' amplitudes in the single trial LFPs were normalized by the Eq. 1

$\Delta V_{\text {norm }}=\left(\Delta V-\Delta V_{\text {min }}\right) /\left(\Delta V_{\text {max }}-\Delta V_{\text {min }}\right)$

where $\Delta V_{\min }$ and $\Delta V_{\min }$ are minimum and maximum amplitudes of the response peak identified from a single cortical depth for each rat.

Figs. 9, 10, 11, 12, 13 show the estimated parameters and depicts the variability of single trial LFPs caused by the different types of anesthesia.
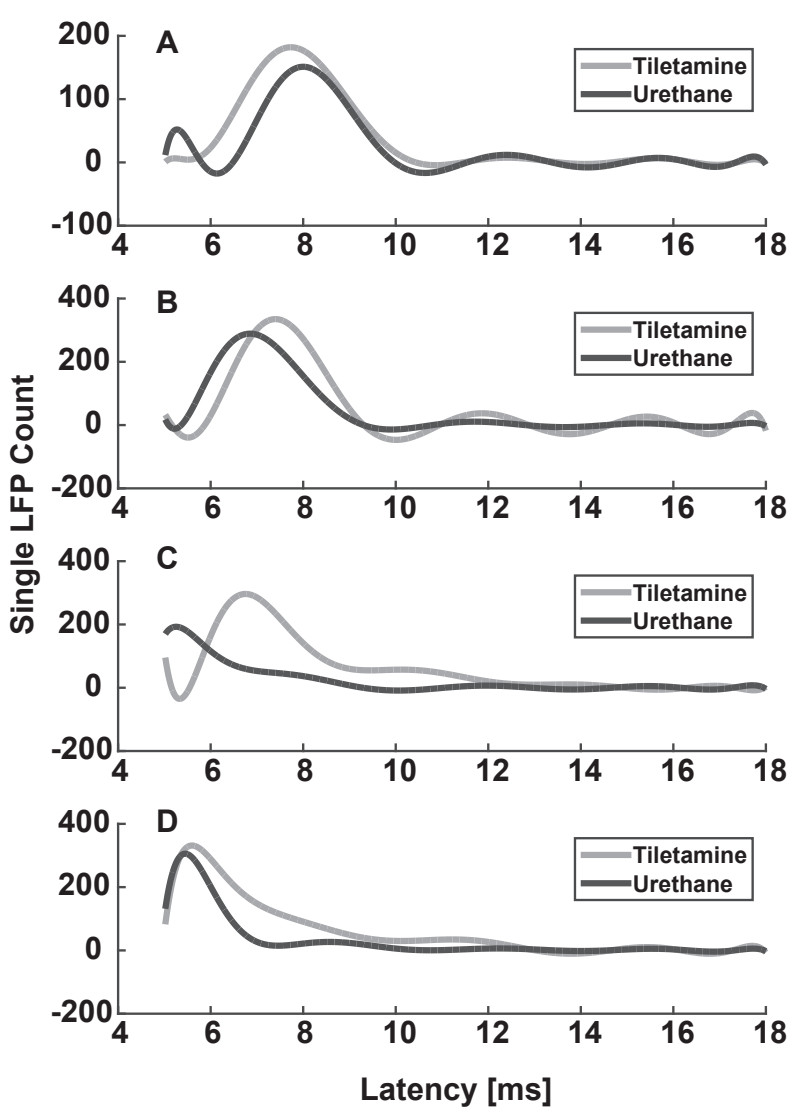

Figure 9 Histogram estimation (bin: $0.45 \mathrm{~ms}$ ) for the $R O L$ at different recording depths. A. $320 \mu \mathrm{m}$ (layer II). B. $420 \mu \mathrm{m}$ (layer III). C. 720 $\mu \mathrm{m}$ (layer IV). D. $920 \mu \mathrm{m}$ (layer Va).

Under Til-Xyl, the evoked responses recorded from the superficial layers (i.e., layer II and III) showed less variability than those recorded from deeper layers (i.e., layer IV and 


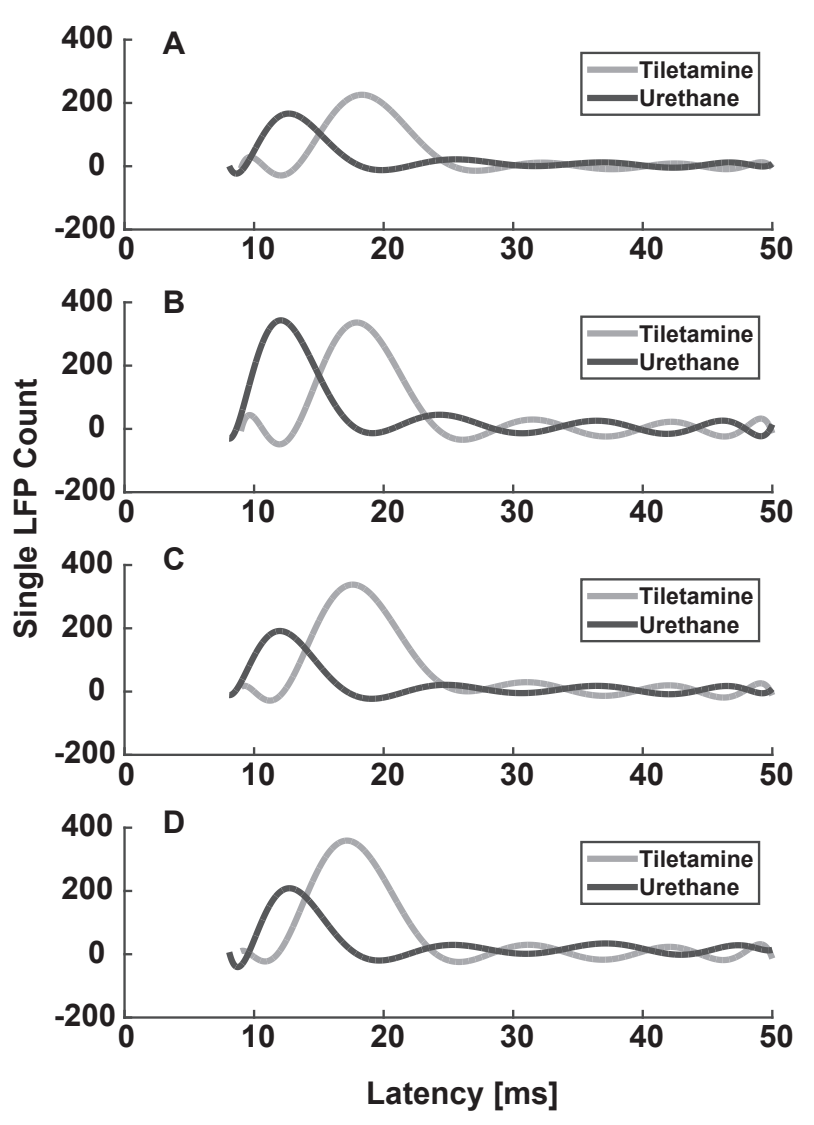

Figure 10 Histogram estimation (bin: $1.25 \mathrm{~ms}$ ) for the $R P L$ at different recording depths. A. $320 \mu \mathrm{m}$ (layer II). B. $420 \mu \mathrm{m}$ (layer III). C. 720 $\mu \mathrm{m}$ (layer IV). D. $920 \mu \mathrm{m}$ (layer Va).

Va). This was highlighted in the distributions of the $R O L$ (Figs. 9 and 114, in gray) and peak latencies (Figs. 10 and $[11 \mathrm{~B}$, in gray): in particular, the distribution of the $R O L$ was wider in the deeper layers than those in the superficial ones.

However, under Urethane, the evoked single trial LFP responses showed different behavior: the distributions of $R O L$ (Figs. 9 and $11 \mathrm{~A}$, in black) and $R P L$ (Figs. 10 and $11 \mathbf{B}$, in black) were slightly narrower in the deeper layers than those in the superficial ones, with the widest distribution in correspondence to layer III. This feature is likely to be associated with different streams of information along the cortical column (modulated by the type and the level of anesthesia) and due to inter-layer connectivity in the barrel network.

The distributions of the $R P L$ were narrower and the $R P$ of the evoked LFPs occurred earlier in the case of Urethane than the ones recorded under Til-Xyl anesthesia. This was confirmed by comparing the mean values of the ROL (Fig. 11 A) and the $R P L$ (Fig. 11 B).

The distributions of the RPA were less informative (Fig. 12), showing wide and flat distributions, however, comparing the mean values of the response-onset amplitude (ROA) and the RPA (Fig. 13), it was noticed that the evoked responses elicited by the whisker stimulation were larger in

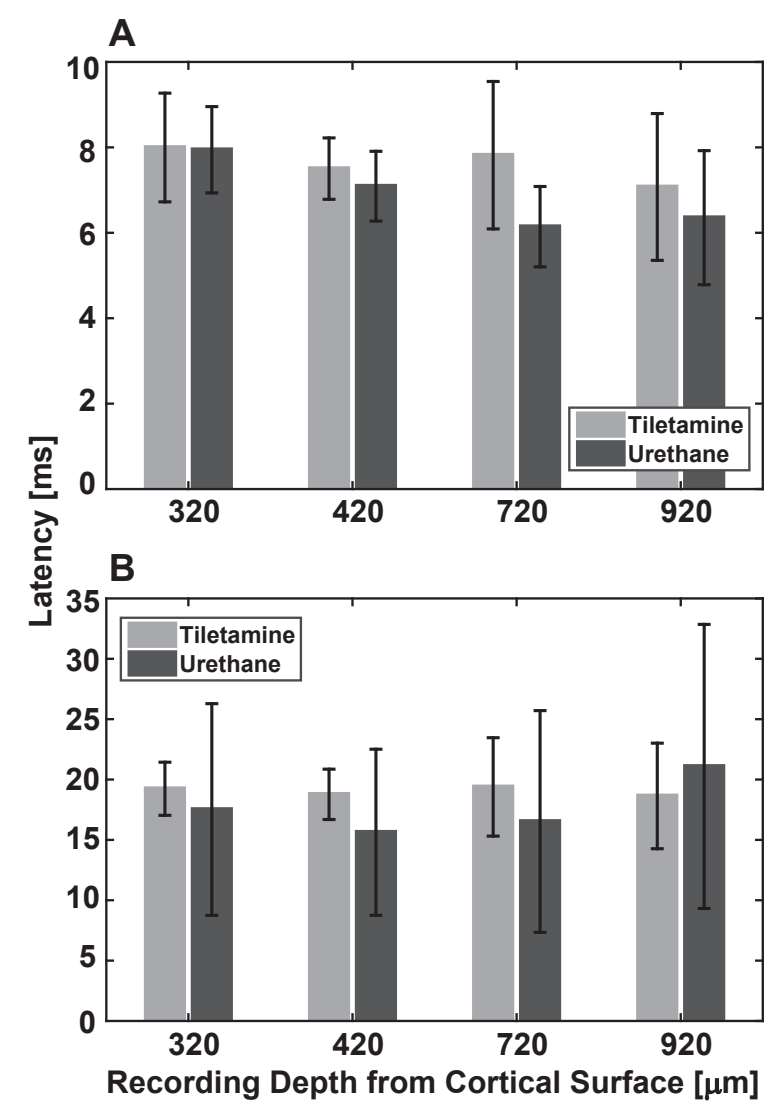

Figure 11 Comparison of the mean latencies for the $R O L(\mathrm{~A})$ and $R P L$ (B). The error bars indicate the standard deviations.

amplitude in animals anesthetized with Urethane. Further experiments are required to understand if this phenomena is associated with diverse effect of the anesthetics on the synaptic plasticity.

\section{Conclusions}

Signals recorded by single neurons are not sufficient to understand brain circuits functions and population approaches are needed. As single LFPs represent fingerprints of a local neuronal population activity, their analysis will help revealing mechanism of information processing by neural networks. The high variability among single trial LFPs poses a challenge in developing general purpose and efficient automatic analysis tools. Moreover, single trial LFP responses are often contaminated by complex stimulation artifacts that must be removed. Therefore the algorithms proposed in this work were designed to perform automatic characterization of evoked single trial LFPs, first by removing the stimulus artifact and then accurately characterizing the various features by quantifying related parameters. As highlighted in the above sections, the tool is efficient and accurate, and capable to capture small differences in the single trial evoked responses. Finally, the tool's purposefulness is demonstrated by its ap- 

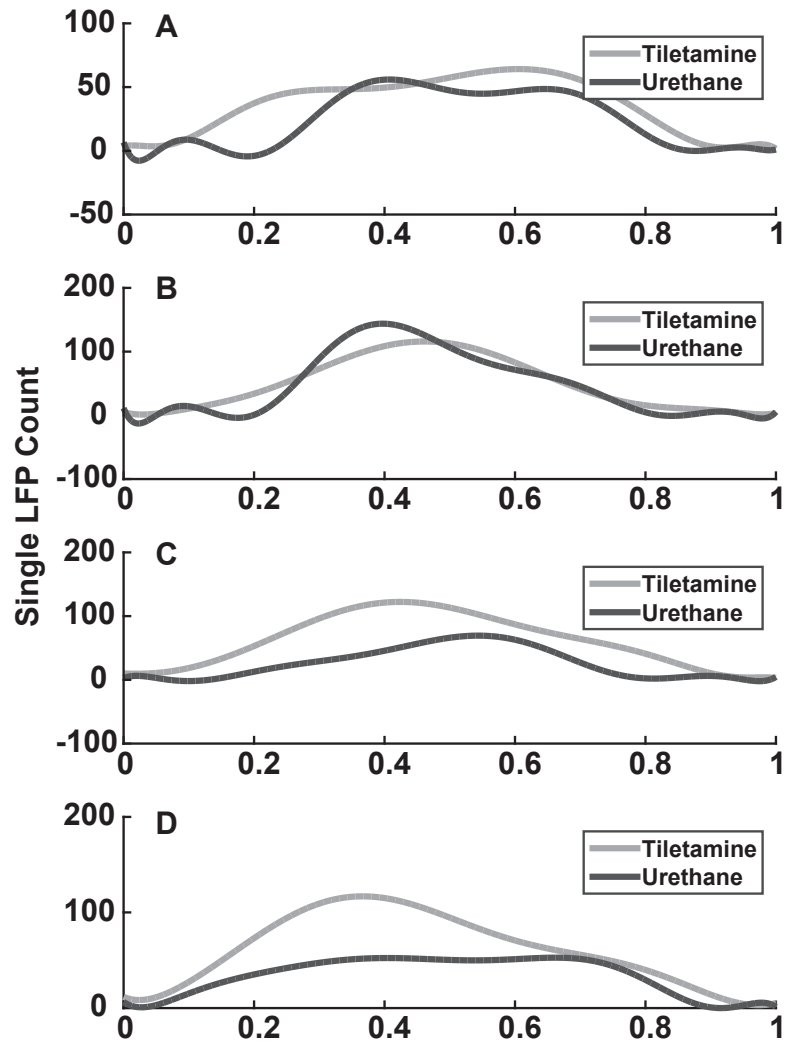

Normalized Amplitude Difference [mV]

Figure 12 Histogram estimation (bin: $1.25 \mathrm{~ms}$ ) for the $R P A$ at different recording depths. A. $320 \mu \mathrm{m}$ (layer II). B. $420 \mu \mathrm{m}$ (layer III). C. 720 $\mu \mathrm{m}$ (layer IV). D. $920 \mu \mathrm{m}$ (layer Va).

plication to the study of the effect of anesthesia on the variability of single trial LFPs from rat barrel cortex.

Acknowledgements Financial support from the 7th Framework Programme of the European Commission through "RAMP"project (www . rampproject.eu) with contract no. 612058 is acknowledged.

Conflict of Interest Statement: The authors declare that the research was conducted in the absence of any commercial or financial relationships that could be construed as a potential conflict of interest.

Authors and Contributors: This work was carried out in close collaboration between all co-authors. SV identified the research objective. MM and SV conceived the method. CC performed the signal acquisition and manual data analysis. MM performed the programming and the automatic data analysis. MM, CC, and SV wrote the paper. All authors have contributed to, seen and approved the final manuscript.

Ethical Approval: All applicable international, national, and institutional guidelines for the care and use of animals were followed.

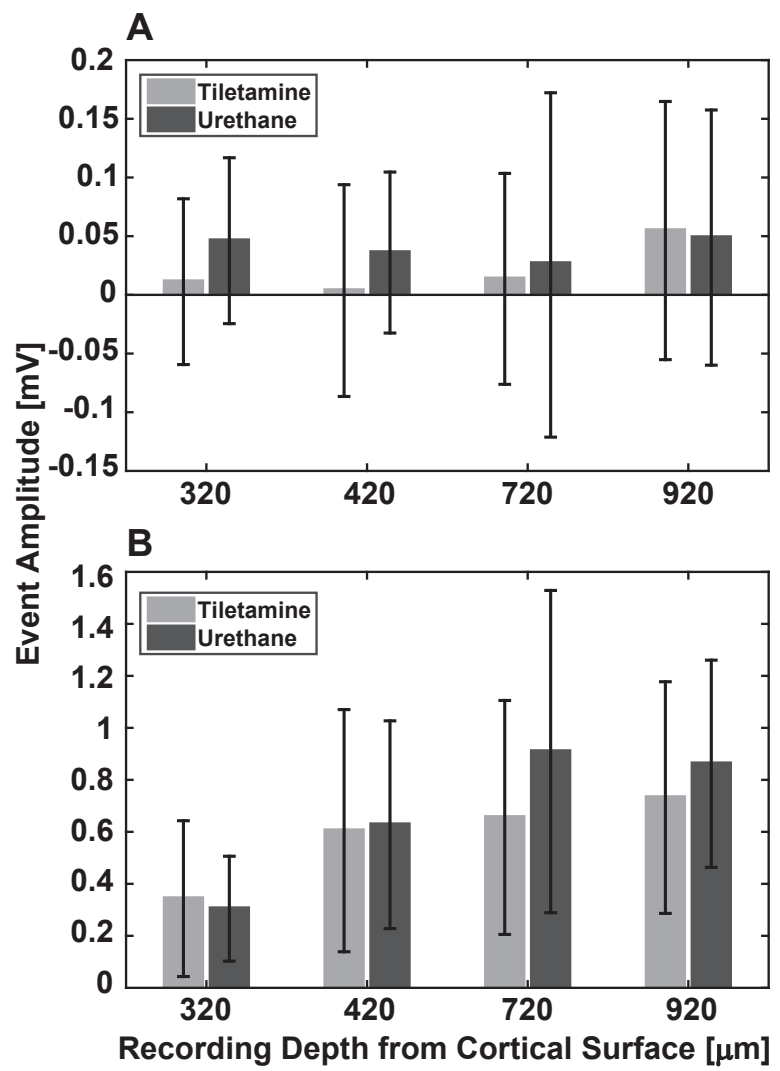

Figure 13 Comparison of the mean amplitudes for the ROA (A) and $R P A$ (B). The standard deviation is represented by the error bars.

\section{References}

1. Ahissar E, Knutsen PM. Object localization with whiskers. Biol Cybern. 2008;98(6):449-458.

2. Diamond ME, von Heimendahl M, Knutsen PM, Kleinfeld D, Ahissar E. 'Where' and 'what' in the whisker sensorimotor system. Nat Rev Neurosci. 2008;9:601612.

3. Schubert D, Kotter R, Staiger JF. Mapping functional connectivity in barrel-related columns reveals layer- and cell type-specific microcircuits. Brain Struct Funct. 2007;212:107-119.

4. Buzski G, Anastassiou CA, Koch C. The origin of extracellular fields and currents - EEG, ECoG, LFP and spikes. Nat Rev Neurosci. 2012; 13(6):407-20.

5. Mahmud M, Pasqualotto E, Bertoldo A, Girardi S, Maschietto M, Vassanelli S. An automated method for detection of layer activation order in information processing pathway of rat barrel cortex under mechanical whisker stimulation. J Neurosci Methods. 2011;196:141150. doi: http://dx.doi.org/10.1016/j. jneumeth.2010.11.024

6. Legatt A, Arezzo J, Vaughan HG. Averaged multiple unit activity as an estimate of phasic changes in local neuronal activity: effects of volume-conducted potentials. J Neurosci Methods. 1980;2(2):203217.

7. van Hemmen J, Ritz R. Neural coding: A theoretical vista of mechanisms, techniques, and applications. In: Andersson SI, editor. Lect Notes Comput Sc: Analysis of Dynamical and Cognitive Systems. Berlin / Heidelberg: Springer; 1995. p. 75119.

8. Kisley MA, Gerstein GL. Trial-to-trial variability and statedependent modulation of auditory-evoked responses in cortex. J Neurosci. 1999;19(23):10451-60. 
9. Okun M, Naim A, Lampl I. The subthreshold relation between cortical local field potential and neuronal firing unveiled by intracellular recordings in awake rats. J Neurosci. 2010;30(12):4440-8.

10. Rey HG, Pedreira C, Quian Quiroga R. Past, present and future of spike sorting techniques. Brain Res Bull. 2015;119(Pt B):106-17.

11. Quian Quiroga R, Atienza M, Cantero JL, Jongsma MLA. What can we learn from single-trial event-related potentials? Chaos Complex Lett. 2007;2:345363.

12. Mahmud M, Bertoldo A, Girardi S, Maschietto M, Vassanelli S. SigMate: a MATLAB-based automated tool for extracellular neuronal signal processing and analysis. J Neurosci Methods, 2012;207:97-112. doi: http://dx.doi.org/10.1016/j. jneumeth.2012.03.009

13. Rey HG, Ahmadi M, Quian Quiroga R. Single trial analysis of field potentials in perception, learning and memory. Curr Opin Neurobiol. 2015;31:148-55.

14. Ahmadi M, Quian Quiroga R. Automatic denoising of single-trial evoked potentials. Neuroimage. 2013;66:672-80.

15. Mahmud M, Girardi S, Maschietto M, Rahman MM, Bertoldo A, Vassanelli S. Slow Stimulus Artifact Removal through Peak-Valley Detection of Neuronal Signals Recorded from Somatosensory Cortex by High Resolution Brain-Chip Interface. IFMBE Proc. 2010;25(4):2062-2065. doi: http://dx.doi.org/ 10.1007/978-3-642-03882-2_547

16. Mahmud M, Girardi S, Maschietto M, Vassanelli S. An Automated Method to Remove Artifacts Induced by Microstimulation in Local Field Potentials Recorded from Rat Somatosensory Cortex. Proc of the 3rd IEEE ISSNIP Biosignals and Biorobotics conference (BRC2012). Manaus, Brazil, January 9-12, 2012. p. 1-5. doi: http://dx.doi.org/10.1109/BRC.2012.6222169

17. Mahmud M, Travalin D, Bertoldo A, Girardi S, Maschietto M, Vassanelli S. A Contour Based Automatic Method to Classify Local Field Potentials Recorded from Rat Barrel Cortex. Proc 5th Cairo Intl Conf Biomed Eng (CIBEC2010). Cairo, Egypt, 16-18 December 2010. p.163-166, doi: http://dx. doi .org/10.1109/CIBEC. 2010.5716087

18. Mahmud M, Travalin D, Bertoldo A, Girardi S, Maschietto M, Vassanelli S. An Automated Classification Method for Single Sweep Local Field Potentials Recorded from Rat Barrel Cortex under Mechanical Whisker Stimulation. J Med Biol Eng. 2012;32(6):397-404. doi: http://dx.doi.org/10.5405/jmbe.923

19. Cecchetto C, Mahmud M, Vassanelli S. Anesthesia Effect on Single Local Field Potentials Variability in Rat Barrel Cortex: Preliminary Results. Conf Proc 37th Annual Intl. Conf. IEEE Eng Med Biol Soc (EMBC2015). Milan, Italy, August 25-29, 2015; p. 4721 4724. doi http://dx.doi.org/10.1109/EMBC. 2015.7319448

20. Swanson LW. Brain maps: structure of the rat brain. 3rd ed. London: Academic Press; 2003.

21. Eckerson WW. Three tier client/server architecture: achieving scalability, performance, and efficiency in client server applications. Open Inf Syst J., 1995;10(1): 3(20).

22. Mahmud M, Bertoldo A, Maschietto M, Girardi S, Vassanelli S. SigMate: A MATLAB-based neuronal signal processing tool. Conf Proc IEEE Eng Med Biol Soc. 2010; p.1352-1355, doi: http:// dx.doi.org/10.1109/IEMBS.2010.5626747

23. Mahmud M, Bertoldo A, Girardi S, Maschietto M, Pasqualotto E, Vassanelli S. SigMate: A Comprehensive Software Package for Extracellular Neuronal Signal Processing and Analysis. Proc 5th Intl IEEE EMBS Conf Neural Eng (NER2011). Cancun, Maxico, Apr. 27-May 1, 2011. p. 88-91, doi: http://dx.doi.org/10.1109/ NER.2011.5910495

24. Ahrens KF, Kleinfeld D. Current flow in vibrissa motor cortex can phase-lock with exploratory rhythmic whisking in rat. J Neurophysiol. 2004;92:1700-7.

25. Kublik E. Contextual impact on sensory processing at the barrel cortex of awake rat. Acta Neurobiol Exp. 2004;64:229-38. 